\title{
Tribocorrosion of passive metals in the mixed lubrication regime: theoretical model and application to metal-on-metal artificial hip joints
}

\author{
Shoufan Cao*, Sandra Guadalupe Maldonado, Stefano Mischler \\ Tribology and Interfacial Chemistry Group, Ecole Polytechnique Fédérale de Lausanne (EPFL), CH-1015 Lausanne, Switzerland
}

\section{A R T I C L E I N F O}

Article history:

Received 5 August 2014

Received in revised form

1 December 2014

Accepted 3 December 2014

Available online 12 December 2014

Keywords:

Tribocorrosion modeling

Mixed lubrication

Wear

Passive metals

Artificial hip joint

\begin{abstract}
A B S T R A C T
Wear is a key problem limiting the lifetime and performance of total hip arthroplasty. The synovial fluid, acting as lubricant, can alleviate the wear of implants but also introduces corrosion. The interaction of mechanical wear and electrochemical corrosion, nominally tribocorrosion, has been proposed as one of the crucial degradation mechanisms of implants and recently saw significant progress in mechanistic understanding and modeling. This study presents a predictive wear model based on a mechanistic approach of tribocorrosion and lubrication and taking into account clinical relevant parameters such as normal load, velocity and clearance. Predicted wear rate corresponds well to the running-in wear rate from simulator experimental results and allows to identify the dominating wear mechanism and to evaluate the influence of several parameters.
\end{abstract}

(c) 2014 Elsevier B.V. All rights reserved.

\section{Introduction}

Hip joints, involving femoral head and acetabular cup, provide the most important movement of the human body. They are wrapped in a capsule, well lubricated and nourished by synovial fluid. Unfortunately, the natural hip joints sometimes loses functionality as a consequence of diseases or accidents. Hip joint replacement is a common clinical solution in such events. There are about 200,000 and 80,000 replacement interventions per year in USA and UK, respectively, and these numbers are estimated to increase by about $170 \%$ by 2030 [1]. Wear is still a major problem causing the failure of hip joint replacement and limiting the longterm performance especially for younger and more active patients who expect a lifetime of artificial hip joints in excess of 20 years.

Essentially, there are two types of hip joint implants based on their tribological features: low friction implants and low wear implants. A metallic or ceramic femoral head in contact with a polymer acetabular cup (MoP or CoP) exhibits low friction, but the softer polymer undergoes usually significant wear. The low wear implants employ Metal on Metal (MoM) or Ceramic on Ceramic $(\mathrm{CoC})$ couples, which suffer of little wear. MoP or CoP is the most used artificial hip joint materials since around the 1960s [2]. However, wear of the polymer releases a large amount of small particles that cause inflammation of the surrounding tissues and

\footnotetext{
* Corresponding author. Tel.: +41216932948.

E-mail address: shoufan.cao@epfl.ch (S. Cao).
}

eventually implants loosening (wear particle disease). In order to reduce the wear of artificial hip joints, MoM (mainly self mated CoCrMo alloys) and $\mathrm{CoC}$ (mainly self mated alumina based ceramics) combinations have been proved to be good alternatives. Wear of MoM or CoC coupling releases nanometer-sized particles and ions that are less prone to initiate particle disease than the micrometer-sized polymer wear debris [3,4]. Although MoM couplings offer a unique combination of wear and impact resistance, the continuous release of metal into the body is of longterm concern as it can cause allergies and other toxic reactions [57]. Material release from MoM artificial hip joints occurs mainly by tribocorrosion of the sliding surfaces which are exposed to the corrosive body fluids $[3,8,9]$. Assessing the lifetime of MoM hip joints requires thus reliable models for predicting the extent of tribocorrosion.

This study aims thus at developing a predictive mechanistic based degradation model of artificial hip joints by combining existing tribocorrosion and lubrication formalisms. For validation, model predictions will be compared to published wear data of MoM pairings. Furthermore, the relevance of different materials, mechanical and geometrical parameters will be evaluated.

\section{Existing approaches for wear prediction of artificial hip joints}

Early wear formalisms were based on the empirical assumption that wear is linearly proportional to the normal load and sliding 


\begin{tabular}{|llll|}
\hline \multicolumn{2}{|l|}{ Nomenclature } & $M$ & Atomic mass $(\mathrm{g} / \mathrm{mol})$ \\
& & $n$ & Oxidation valence \\
$U_{\text {tot }}$ & Total wear volume $\left(\mathrm{mm}^{3}\right)$ & $F$ & Faraday's constant $(\mathrm{C} / \mathrm{mol})$ \\
$U_{\text {mech }}$ & Mechanical wear volume $\left(\mathrm{mm}^{3}\right)$ & $\rho$ & Density $\left(\mathrm{g} / \mathrm{cm}^{3}\right)$ \\
$U_{\text {chem }}$ & Chemical wear volume $\left(\mathrm{mm}^{3}\right)$ & $h_{\text {min }}$ & Minimum film thickness $(\mathrm{nm})$ \\
$V_{\text {tot }}$ & Total wear rate $\left(\mathrm{mm}^{3} / \mathrm{s}\right)$ & $u$ & Entraining velocity $(\mathrm{mm} / \mathrm{s})$ \\
$V_{\text {mech }}$ & Mechanical wear rate $\left(\mathrm{mm}^{3} / \mathrm{s}\right)$ & $\omega$ & Angular velocity $(\mathrm{rad} / \mathrm{s})$ \\
$V_{\text {chem }}$ & Chemical wear rate $\left(\mathrm{mm}^{3} / \mathrm{s}\right)$ & $\eta$ & Viscosity of solution $(\mathrm{Pa} \cdot \mathrm{s})$ \\
$U_{\text {running-in }} \quad$ Running-in wear volume $\left(\mathrm{mm}^{3}\right)$ & $E$ & Young's modulus $(\mathrm{GPa})$ \\
$k_{\text {mech }} \quad$ Proportionality factor for mechanical wear & $E^{\prime}$ & Effective Young's modulus $(\mathrm{GPa})$ \\
$k_{\text {chem }}$ & Proportionality factor for chemical wear & $\mathrm{R}$ & Radius of curvature $(\mathrm{mm})$ \\
$F_{\mathrm{n}}$ & Normal force $(\mathrm{N})$ & $R^{\prime}$ & Effective radius of curvature $(\mathrm{mm})$ \\
$F_{\text {eff }}$ & Effect normal force carried by asperities $(\mathrm{N})$ & $c_{\mathrm{R}}$ & Radial clearance $(\mathrm{mm})$ \\
$H$ & Micro surface hardness $(\mathrm{HV})$ & $\nu$ & Poisson's ratio \\
$L$ & Sliding distance $(\mathrm{mm})$ & $R_{\mathrm{a}}$ & Surface roughness $(\mu \mathrm{m})$ \\
$V_{\mathrm{s}}$ & Sliding velocity $(\mathrm{mm} / \mathrm{s})$ & $k_{0}$ & Proportionality factor for effective load \\
$I_{\mathrm{P}}$ & The excess current $(\mathrm{mA})$ & $\mathrm{SHE}$ & Standard hydrogen electrode \\
$Q_{\mathrm{P}}$ & Passivation charge density $\left(\mathrm{mC} / \mathrm{cm}^{2}\right)$ & & \\
\hline
\end{tabular}

distance $[2,10,11]$. These formalisms are in agreement with Archard's theorem implying a proportionality between wear and the real area of contact established at plastically deforming asperity junctions and the sliding distance. The real area of contact is given by the ratio of normal load to indentation hardness, Predictions from such models were found to correlate well with experimental data obtained from hip joint simulators for both the running-in (the initial operation period characterized by relatively high wear rates) and the steady state (subsequent low wear rate period) wear regimes $[10,11]$. However, the limited number of operational parameters considered in Archard's formalism does not allow to explain the effect of other factors such as the clearance that play a crucial role on wear of MoM pairings [12].

The environment of the artificial hip joints in human body is synovial fluid, which is a complex water based solution containing many different kinds of proteins, organic molecules, as well as cells and aggressive salts such as $\mathrm{NaCl}$ [13]. On one hand, synovial fluid acts as lubricants, alleviating the wear of artificial hip joints and on the other hand synovial fluid provides a corrosive medium causing tribocorrosion damage of the implant metals.

The effect of elastohydrodynamic lubricating films on wear of MoM implants was addressed by Dowson [14-16]. He postulated that wear occurs mainly during the mixed lubrication regimes of gait where the load is carried out partially by asperity contacts and partially by the hydrodynamic fluid film. Increasing fluid film thickness reduces the load carried out by asperities and thus it is supposed to reduce wear. Indeed, Dowson [15] found out by comparing a large amount of data of wear results from simulators that the extent of wear is inversely proportional to the hydrodynamic fluid film thickness as calculated using elastohydrodynamic models (Hamrock-Dowson equation).

Boundary lubrication is also affected by the presence of body fluids. The interaction of synovial fluid with the bearing CoCrMo surfaces may lead to the formation of carbonaceous tribolayers formed by aggregated [13] or denatured proteins $[8,17]$ that provide boundary lubrication. Unfortunately, up to date no formalism quantitatively relating wear to boundary lubrication is available.

Tribocorrosion is a material degradation or transformation induced by simultaneous action of wear and corrosion. Friction and wear may modify the sensitivity of material to corrosion and conversely corrosion may modify the conditions of friction. CoCrMo alloys used in artificial hip joints spontaneously develop in aqueous environments a thin surface oxide layer (passive layer), which acts as a barrier against the release of ions. However, wear depassivates the metal surface by damaging the passive layer and thus exposes the much more reactive bare metal to the solution, causing metal oxidation and dissolution until the passive film forms again (repassivation). Repeated depassivation/repassivation cycles results in wear accelerated corrosion. Clear evidence of wear and corrosion has been detected in explants $[3,18]$ where metal debris was manifestly generated by the combined effect of mechanics and chemistry. Tribocorrosion has been the object of several studies aimed at elucidating the interaction mechanisms of wear and corrosion and at developing predictive models for describing material degradation induced by synergistic effects of wear and corrosion [19]. A general model was proposed by Uhlig [20] for fretting corrosion based on two distinct mechanisms: mechanical wear and wear accelerated corrosion. Then Mischler et al. [21-23] applied the model to sliding tribocorrosion and further studied the interaction of mechanical wear and electrochemical corrosion. According to those studies, the total wear $U_{\text {tot }}$ removed by tribocorrosion is given by Eq. (1):

$U_{\text {tot }}=U_{\text {mech }}+U_{\text {chem }}$

where $U_{\text {mech }}$ is mechanical wear and $U_{\text {chem }}$ is chemical wear. $U_{\text {chem }}$ can be further subdivided into two parts: the wear accelerated corrosion inside the wear track and the corrosion outside the wear track. Since the former one is much larger comparing to the latter one, normally only the wear accelerated corrosion is considered in the calculation of chemical wear. Several models [24] are available for describing wear accelerated corrosion as a consequence of plastic deformation at asperity contacts. These models predict a proportionality between wear accelerated corrosion rate and the ratio of load to hardness at a power ranging from 0.5 (rough surface sliding against smooth counterpart) or 1 (both counterparts rough), the sliding velocity and the passivation charge density, that is, the amount of anodic charge (corresponding to the amount of oxidized metal) needed to repassivate the bare metal exposed to the corrosive environment. Such models were found to accurately describe the tribocorrosion behaviour of a CoCrMo alloy under idealized laboratory conditions (inert ceramic counter, sulphuric acid as model electrolyte, high contact pressure exceeding the plastic yield of the alloy, stroke frequency lower than a few $\mathrm{Hz}$ in order to allow sufficient time for repassivation between two strokes) [25]. Moreover, these models were tentatively applied to the MoM contact conditions and found correlating well with the wear rates reported in literature for MoM couplings tested in simulators [9]. According to this study, wear 
accelerated corrosion is likely to account for a significant fraction of the overall MoM degradation in clinical relevant conditions. Tribocorrosion models do not however consider the lubrication, which constitutes, as demonstrated by Dowson [14,15], a crucial factor for the wear of MoM pairings.

\section{Tribocorrosion model for mixed lubrication}

Fig. 1 illustrates the physical model underlying the present attempt to model tribocorrosion in mixed lubricated regimes characteristics of relatively smooth surfaces as found in hip joints. Direct contact between the two solid surfaces occurs only locally at asperity junctions (Fig. 1a) or contacts between asperity and flat part of the counter body (Fig. 1b). Such contacts are supposed to behave plastically. Thus, when the two bodies move with respect to each other, at the contact spots, mechanical wear and wear accelerated corrosion may occur.

Mechanical wear can be described by the Archard's theorem relating the extent of wear to the contact area established by plastic deformation at the contact spots. The sum of the contact areas of all spots corresponds to the ratio between normal load $F_{\mathrm{n}}$ and surface hardness $H$ and thus the wear rate can be described by Eq. (2) that relates volumetric wear $U_{\text {mech }}$ as a function of $F_{\mathrm{n}}, H$ and sliding distance $L$ with $k_{\mathrm{m}}$ as proportionality factor:

$U_{\text {mech }}=k_{m}\left(\frac{F_{n}}{H}\right) L$

The wear rate can be defined by introducing the average sliding velocity $v_{\mathrm{s}}$ according to Eq. (3):

$V_{\text {mech }}=k_{\mathrm{m}}\left(\frac{F_{\mathrm{n}}}{H}\right) v_{\mathrm{s}}$

Liu et al. [10] and Uddin et al. [11] used such an equation to predict wear rate and wear distribution of MoM artificial hip joints. The former study identified two wear coefficients $k_{\mathrm{m}}$ of a resurfacing CoCr alloy hip joint: $1.13 \times 10^{-8} \mathrm{~mm}^{3} /(\mathrm{N} \cdot \mathrm{m})$ for short-term, up to one million gait cycles and $1.2 \times 10^{-9} \mathrm{~mm}^{3} /(\mathrm{N} \cdot \mathrm{m})$ for long-term, up to 50 million gait cycles, respectively. The later obtained $0.5 \times 10^{-8}$ and $1.5 \times 10^{-9} \mathrm{~mm}^{3} /(\mathrm{N} \cdot \mathrm{m})$ for running-in and steady state period respectively for a CoCrMo alloy hip joint.

Studies of wear accelerated corrosion of passive metals are usually carried out by imposing a passive electrode potential to the metal while rubbing against a counter piece. In this way, the acceleration of corrosion can be measured by recording the excess current necessary to maintain the passive potential during rubbing. The excess current can be related to the material loss through
Faraday's law. Models exist for describing the extent of anodic current induced by sliding on passive metals [22,24]. Such models consider that asperity interactions with the counter body either leave behind deformed and depassivated asperities (Fig. 1a) or a track of depassivated, abraded metal (Fig. 1b). In both situations, the excess current $I_{\mathrm{p}}$ can be described [24] by Eq. (4):

$I_{\mathrm{P}}=k_{\mathrm{c}} Q_{\mathrm{P}} v_{\mathrm{S}}\left(F_{\mathrm{n}} / H\right)^{0.5}$

where, $k_{\mathrm{c}}$ is a proportionality factor, $Q_{P}$ is the passivation charge density, and $v_{\mathrm{s}}$ is the sliding velocity. Details of this model can be found in references [22 and 24].

Then chemical wear model can be obtained by integrating the repassivation current model (Eq. (4)) into Faraday's law:

$V_{\text {chem }}=\frac{I_{\mathrm{P}} M}{n F \rho}=\frac{k_{\mathrm{c}} Q_{\mathrm{P}} M v_{\mathrm{s}}\left(F_{\mathrm{n}} / H\right)^{0.5}}{n F \rho}$

where, $M$ is the atomic mass of the metal, $n$ is oxidation valence, $F$ is Faraday's constant (approximately 96,500 C/mol), and $\rho$ is the density.

Eqs. (2) and (4) were developed for situation of boundary lubricated contacts where the thickness of the hydrodynamic films is too small to provide any load bearing capacity and effective separation of the two contacting bodies. In the case of mixed lubricated contacts, part of the load $F_{\mathrm{n}}$ is carried out by the hydrodynamic fluid film and only a fraction of it acts as effective force $F_{\text {eff }}$ at asperity contacts which generates mechanical wear and wear accelerated corrosion. The extent of $F_{\text {eff }}$ depends on one hand on the thickness of the hydrodynamic film and on the other hand on the surface roughness.

Hip joints operate for most of the time in mixed lubrication conditions and therefore part of the load is carried out by the hydrodynamic fluid film and only a fraction of it generates wear. Indeed, Dowson [15] found an empirical correlation between a set of wear results obtained by testing a variety of CoCrMo MoM hip joints in simulators and the corresponding theoretical minimum hydrodynamic film thickness in the running-in period.

$U_{\text {running-in }}=\frac{93.97}{\left(h_{\min }\right)^{1.49}}$

The experimental data have a large scattering due to the differences in simulator conditions, materials and calf serum additives since they are results from eight laboratories in the UK, Canada and USA. However, the broad data set is thought to give an accurate enough picture of the relationship between running-in wear and film thickness. The minimum film thickness $h_{\min }$ was a

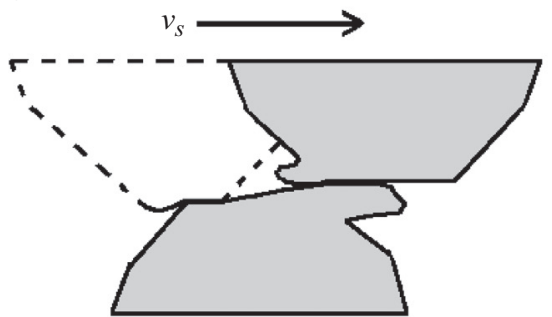

b

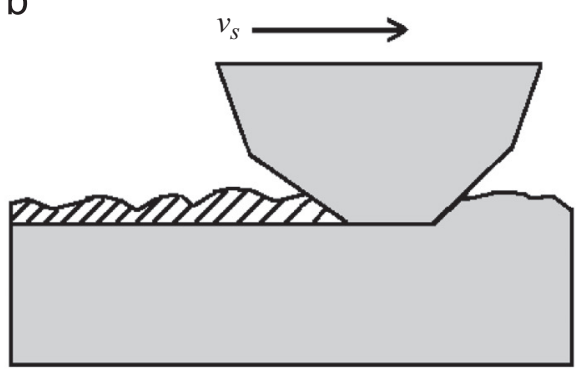

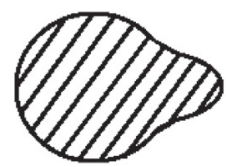

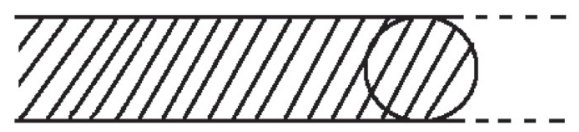

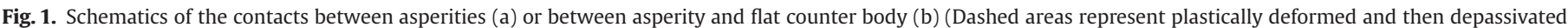
metal surface). 
theoretically calculated using the Hamrock-Dowson equation [26]:

$h_{\min }=2.8\left(\frac{u \eta}{E^{\prime} R^{\prime}}\right)^{0.65}\left(\frac{F_{\mathrm{n}}}{E^{\prime} R^{\prime 2}}\right)^{-0.21} R^{\prime}$

where, $u$ corresponds to the entraining velocity and for hip joints: $u=(R \omega+0) / 2=R \omega / 2, \eta$ is the viscosity of solution, $w$ is the normal load, $E^{\prime}$ is the effective Young's modulus, $2 / E^{\prime}=\left(1-v_{1}^{2}\right) / E_{1}+\left(1-v_{2}^{2}\right) / E_{2}$, and $R^{\prime}$ is the effective radius of curvature, $1 / R^{\prime}=1 / R_{1}+1 / R_{2}$ (for convex surfaces), $1 / R^{\prime}=1 / R_{1}-1 / R_{2}$ (for concave surfaces).

The empirical Eq. 6 predicts lubricated wear of artificial hip joints being inversely proportional to the minimum film thickness to the power of 1.49 . The wear reducing effect of hydrodynamic lubrication is ascribed to the reduction in load effectively carried out by the contacting asperities [15]. Thus the ratio between the load carried out by asperity contacts (effective normal load $F_{\text {eff }}$ ) and the overall load applied to the contact $\left(F_{\mathrm{n}}\right)$ in mixed lubrication regime is inversely proportional to the film thickness at the power 1.49. This relation is of course valid for the situation of CoCrMo MoM hip joints characterised by specific mechanical alloy properties and polished surfaces characterised by typical $R_{\mathrm{a}}$ value of $10 \mathrm{~nm}$.

Then, the overall load can be corrected by film thickness in order to get the effective load in mixed lubrication regimes:

$F_{\text {eff }}=k_{0} \frac{F_{\mathrm{n}}}{\left(h_{\min }\right)^{1.49}}$

Introducing $F_{\text {eff }}$ as the effective load responsible for wear and depassivation of asperities into Eqs. (3) and (4) and combining with Eq. (1) yields a general equation for the tribocorrosion degradation:

$V_{\text {tot }}=k_{\mathrm{m}}\left(\frac{k_{0} F_{\mathrm{n}} / h_{\min }^{1.49}}{H}\right) v_{\mathrm{s}}+k_{\mathrm{c}} \frac{Q_{\mathrm{P}} M v_{\mathrm{s}}\left(\frac{k_{0} F_{\mathrm{n}} / h_{\min }{ }^{1.49}}{H}\right)^{0.5}}{n F \rho}$

An explicit composite model can be obtained by adding the minimum film thickness equation (Eq. (7)):

$V_{\text {tot }}=k_{\text {mech }} \frac{\left(E^{\prime}\right)^{0.6556}}{\eta^{0.9685}} \cdot \frac{\left(F_{n}\right)^{1.3129}\left(v_{s}\right)^{0.0315}}{\left(R^{\prime}\right)^{1.1473} H}+k_{\text {chem }} \frac{M Q_{P}\left(E^{\prime}\right)^{0.3278}}{n F \rho \eta^{0.4843}} \cdot \frac{\left(F_{n}\right)^{0.6565}\left(v_{s}\right)^{0.5158}}{\left(R^{\prime}\right)^{0.5737} H^{0.5}}$

where, $k_{\text {mech }}$ is the mechanical wear factor, $k_{\text {mech }}=k_{\mathrm{m}} k_{0} /\left(2.8^{1.49}\right)$, while $k_{\text {chem }}$ is the chemical wear factor, $k_{\text {chem }}=k_{\mathrm{c}} k_{0}^{0.5} /\left(2.8^{0.745}\right)$.

The composite model relates wear to mechanical, electrochemical, material and fluid parameters. The proportionality factors $k_{\text {mech }}$ and $k_{\text {chem }}$ are system specific and require appropriate experimental calibration.

\section{Model calibration}

Tribometer experimental results from reference [25] are used to calibrate the model. In this work, the tribocorrosion of a biomedical CoCrMo alloy sliding against inert alumina was investigated in sulphuric acid under different applied passive potentials and different loads. The chemical and mechanical wear volumes were determined by integrating the excess anodic current during rubbing and by subtracting $U_{\text {chem }}$ to $U_{\text {tot }}$ (see Eq. (1)), respectively. The parameters used in [25] are listed in Table 1.

The passivation charge density was measured by separate electrochemical experiments for each of the investigated potentials. The hardness of the metal was measured inside and outside of the wear track and revealed considerable work hardening due to friction. Therefore the hardness measured inside of the wear track was considered for evaluation.
Table 1

Tribocorrosion experimental parameters from [25].

\begin{tabular}{ll}
\hline Movement & Reciprocating sliding \\
Configuration & Ball $\left(\mathrm{Al}_{2} \mathrm{O}_{3}, R=3 \mathrm{~mm}\right)$ on disk (CoCrMo alloy) \\
Normal forces/N & $1.1,5.8,11.7,17.5$ \\
Stroke length $/ \mathrm{mm}$ & 5 \\
Frequency $/ \mathrm{Hz}$ & 1 \\
Velocity $/(\mathrm{mm} / \mathrm{s})$ & 20 \\
Applied potentials $/ \mathrm{V}_{\text {SHE }}$ & Passive: $0.254,0.654,1.054$ \\
Lubricant $($ Electrolyte $)$ & $0.5 \mathrm{M} \mathrm{H}_{2} \mathrm{SO}_{4}$ \\
Testing time $/ \mathrm{s}$ & 1800 \\
\hline
\end{tabular}

According to Eq. (10), linear dependence of mechanical and chemical wear with the factors $F_{\mathrm{n}}^{1.3129} / H$ and $Q_{\mathrm{p}} F_{\mathrm{n}}^{0.6565} / H^{0.5}$ are expected for the data from reference [25] This is indeed observed in Fig. 2, in particular for the chemical wear. Note that the experimental data are subject to some scattering partly related to uncertainties in the quantification of wear using profilometry and the current, as discussed in more details in references [19,27].

The slopes of the graphs in Fig. 2 allow one to extract the $k_{\text {mech }}$ and $k_{\text {chem }}$ values according to Eq. (10) and the experimental parameters listed in Tables 1 and 2.

By determining the constant factors for the mechanical wear rate $k_{\text {mech }}$ and chemical wear rate $k_{\text {chem }}$ the composite model can thus be calibrated and more precisely described by Eq. (11):

$$
\begin{aligned}
V_{\text {tot }}= & 0.01363 \frac{\left(E^{\prime}\right)^{0.6556}}{\eta^{0.9685}} \cdot \frac{\left(F_{\mathrm{n}}\right)^{1.3129}\left(v_{\mathrm{s}}\right)^{0.0315}}{\left(R^{\prime}\right)^{1.1473} H} \\
& +750.35 \frac{M Q_{\mathrm{P}}\left(E^{\prime}\right)^{0.3278}}{n F \rho \eta^{0.4843}} \cdot \frac{\left(F_{\mathrm{n}}\right)^{0.6565}\left(v_{\mathrm{s}}\right)^{0.5158}}{\left(R^{\prime}\right)^{0.5737} H^{0.5}}
\end{aligned}
$$

The composite model involves a number of material, mechanical and electrochemical parameters that are seldom fully reported in tribocorrosion studies. Especially, hardness after sliding, solution viscosity and passivation charge density are often been omitted although being important variables. Passivation charge density $\left(Q_{P}\right)$ is the charge per surface area needed to form the passive film from the bare metal or reform the passive film when the passive film is abraded. So, it represents the charge flow during the depassivation and repassivation. $Q_{p}$ can be determined by independent electrochemical experiments where the charge is measured by integrating the current density when switching the applied potential from cathodic to a given passive value. This method is based on two assumptions. On one hand the cathodic partial current should be negligible in order to make the measured current equal to the metal oxidation current. This is usually fulfilled for anodic polarization in the passive domain [24]. On the other hand the oxide film should be removed under the cathodic potential. Bardwell et al. [28,29] found that the oxide film of iron and iron-chromium alloys can be removed by cathodic polarization in acid solutions but not quite so efficiently in neutral solutions. Other sources of uncertainties are discussed elsewhere [22]. Guadalupe et al. [25] use this method to measure the passivation charge density of CoCrMo alloy in $0.5 \mathrm{M}$ sulphuric acid for three different potentials. Hodgson et al. [30] studied the passive behaviour of CoCrMo alloy in buffered $0.14 \mathrm{M} \mathrm{NaCl}$ and gave the passivation charge density under two applied potentials. Fig. 3 shows the data from these two studies. What is interesting is that the passivation charge densities correlate well with the potential but are less affected by the solution's chemical property and the $\mathrm{pH}$.

Igual Munoz et al. [31,32] studied tribocorrosion of a CoCrMo alloy $\left(\mathrm{Al}_{2} \mathrm{O}_{3}\right.$ ball on CoCrMo disk, normal load: $5 \mathrm{~N}$, velocity: $18.85 \mathrm{~mm} / \mathrm{s}$, ball radius: $3 \mathrm{~mm}$ ) in $0.14 \mathrm{M} \mathrm{NaCl}$ under two different passive 
a

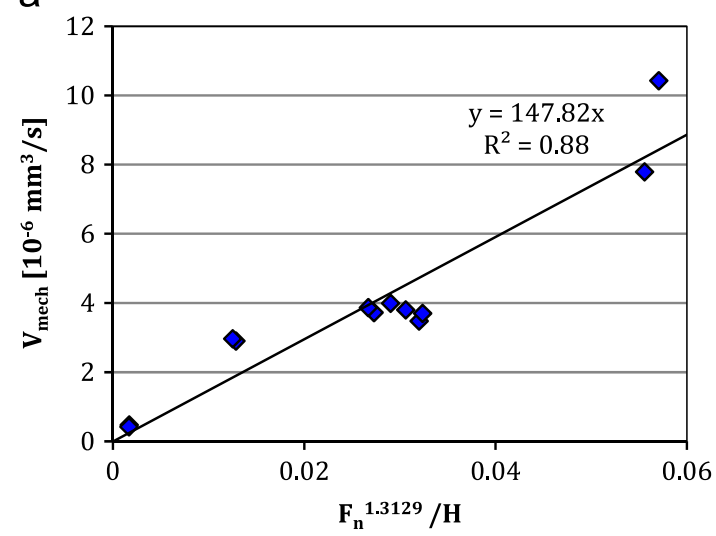

b

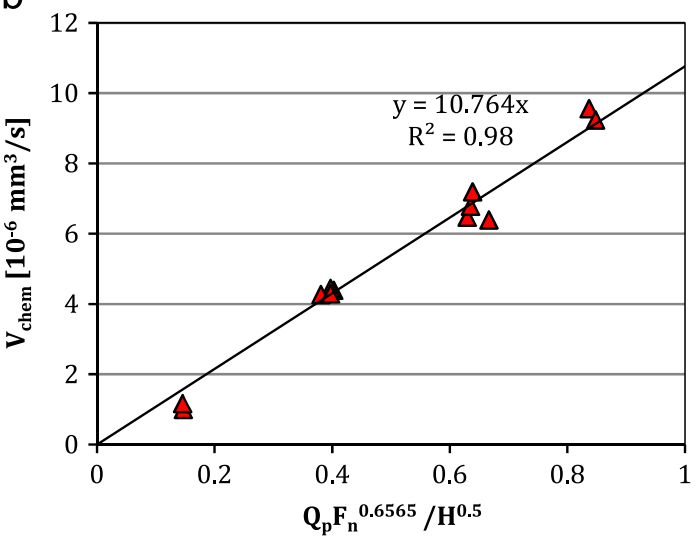

Fig. 2. The correlation between mechanical and chemical wear rate and the group of factors according to the composite model.

Table 2

Constant values for the wear calculations for [25].

\begin{tabular}{ll}
\hline Materials & $\mathrm{Al}_{2} \mathrm{O}_{3}: E=350 \mathrm{GPa}, v=0.22 ; \mathrm{CoCrMo}: E=248 \mathrm{GPa}, v=0.3$ \\
$\mathrm{H}_{2} \mathrm{SO}_{4}$ viscosity & $0.001 \mathrm{~Pa} \cdot \mathrm{s}$ \\
Charge number $(n)$ & $2.37(\mathrm{Co} 2 \times 63 \%, \mathrm{Cr} 3 \times 27 \%$ Mo $5 \times 6 \%)$ \\
$\begin{array}{c}\text { Molecular mass } \\
(M)\end{array}$ & $58.55 \mathrm{~g} / \mathrm{mol}$ \\
Density $(\rho)$ & $8.33 \mathrm{~g} / \mathrm{cm}^{3}$ \\
\hline
\end{tabular}

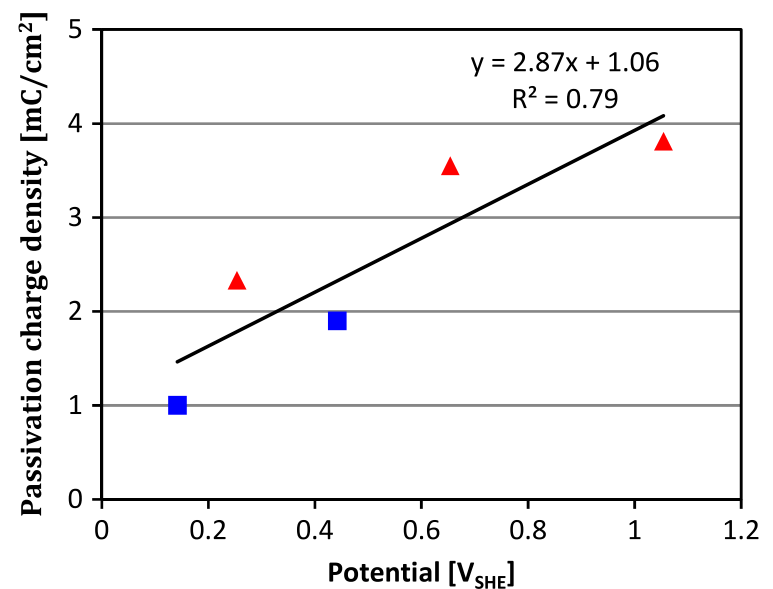

Fig. 3. Passivation charge density at different potentials (Triangular dots from [25] and square dots from [30]).

potentials ( 0.25 and $\left.0.7 \mathrm{~V}_{\mathrm{SHE}}\right)$ and determined the mechanical and chemical wear. The CoCrMo alloy was submitted to different heat treatments in order to alter its microstructure. The surface hardness of the CoCrMo alloy inside the wear track was about $500 \mathrm{HV}$ with only little difference depending on heat treatment. Work hardening was also reported in this study. The passivation charge density was not measured in these two studies but can be extrapolated from the fitting line in Fig. 3 since the potential is known (this yields 1.8 and $3.1 \mathrm{mC} / \mathrm{cm}^{2}$ for potentials of 0.25 and $0.7 \mathrm{~V}_{\mathrm{SHE}}$, respectively). In this case, all of the required parameters are available and thus Eq. (11) can be used to theoretically predict mechanical and chemical wear for the system described in $[31,32]$. The predicted wear and experimental data are shown in Fig. 4.

The predicted chemical and mechanical wear values relatively well represent the two groups of experimental data. This indicates that the calibration of the $k_{\text {chem }}$ and $k_{\text {mech }}$ values achieved in sulphuric acid allows the model to be applied with reasonable precision to other environments. A crucial factor here is the exact

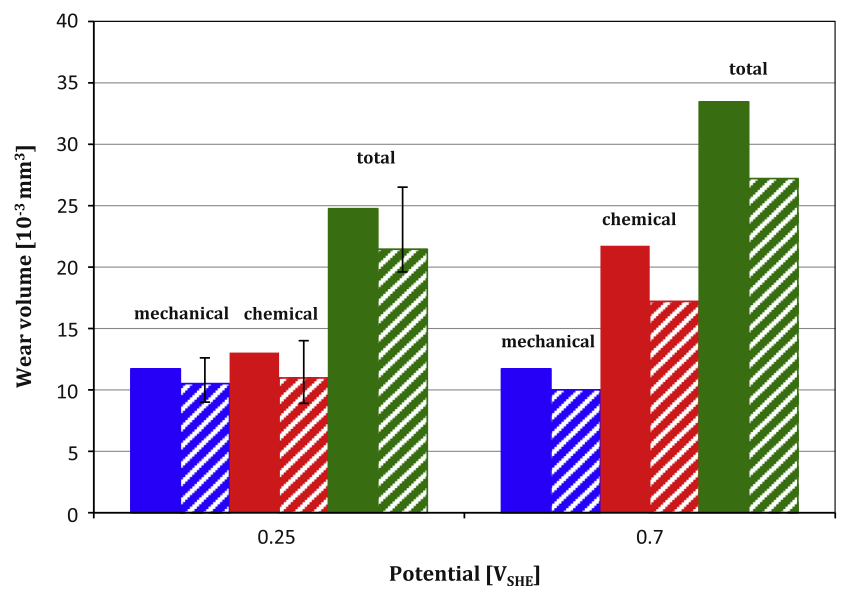

Fig. 4. The predicted mechanical, chemical and total wear (solid columns) at two potentials under tribometer conditions $\left(\mathrm{Al}_{2} \mathrm{O}_{3}\right.$ ball on CoCrMo disk, normal load: $5 \mathrm{~N}$, velocity: $18.85 \mathrm{~mm} / \mathrm{s}$, ball radius: $3 \mathrm{~mm}$ ) and experimental data (dashed columns) from [31] and [32].

determination of the prevailing electrochemical conditions and of the corresponding passivation charge density.

\section{Model predictions}

\subsection{Instantaneous wear rate during one gait cycle}

Tribometers operate usually under constant normal load and velocity and thus the wear rate should not vary with time. In order to mimic the movement of hip joints, simulators adopt fluctuant normal load and velocity. Thus variations of the wear rate are expected to occur during a gait cycle. The composite model allows estimating these variations during a single gait cycle, as shown in Fig. 5b. A self mated CoCrMo contact is considered and relevant parameters are listed in Table 2. Sliding velocity is calculated by multiplying the radius of the head by flexion-extension angular velocity given by the ISO standard 14242-1:2012. A surface hardness of $700 \mathrm{HV}$ for CoCrMo alloys after work hardening and a fluid viscosity of $0.001 \mathrm{~Pa} \cdot \mathrm{s}$ are assumed. The composite radius can be calculated using the head radius and clearance. Details are described in reference [15]. Studies [33,34] found that the OCP of CoCrMo in hip joint simulators varied from -0.1 to $0.1 \mathrm{~V}_{\mathrm{SHE}}$ that corresponds to an approximate passivation charge density of $1 \mathrm{mC} / \mathrm{cm}^{2}$ (Fig. 3). The wear rate predicted by the model under these conditions during a gait cycle is illustrated in Fig. 5 together 
a

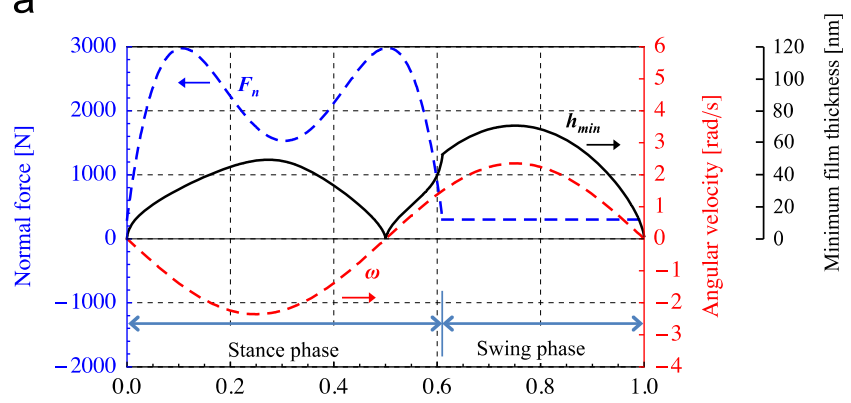

b

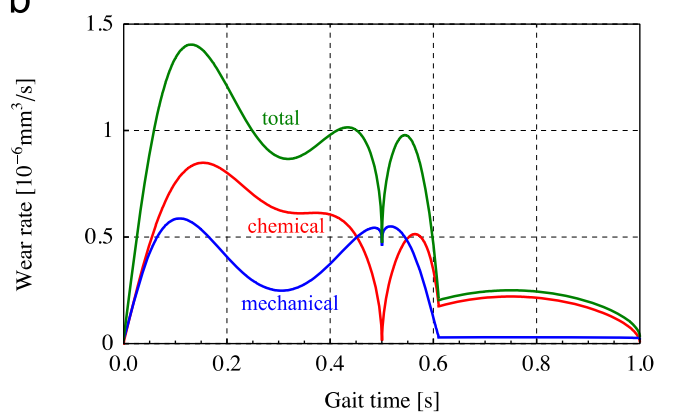

Fig. 5. Evolution with time of normal load $\left(F_{n}\right)$, flexion-extension angular velocity $(\omega)$ according to ISO 14242-1:2012 and calculated minimum film thickness $\left(h_{\min }\right)$ in figure (a) and calculated mechanical, chemical and total wear rate in figure (b) (CoCrMo alloy, $R=18 \mathrm{~mm}, c_{\mathrm{R}}=30 \mu \mathrm{m}$ ).

with the evolution of normal force, angular velocity and film thickness as calculated according to Eq. (7).

From Fig. 5 it appears that both mechanical and chemical wear contribute significantly to the overall wear. The evolution of mechanical wear with time follows the shape of the normal force as expected from Archard's law while the chemical wear rather depends on the instantaneous combination of velocity and normal force. The integrated wear volume in the stance and swing phase is about 0.70 and $0.08 \mathrm{~mm}^{3}$, respectively. The later value is about ten percent of the total wear. As a consequence, although wear is mainly concentrated in the stance phase, significant wear occurs also during the swing phase and this essentially due to wear accelerated corrosion.

\subsection{Predicted running-in wear}

From the instantaneous wear rate in Fig. 5, the wear volume in one second (or in one gait cycle) can be obtained by integrating the wear rate curves shown in Fig. 5 from 0 to $1 \mathrm{~s}$. The representative time of the running-in period in simulator is about one million cycles, which is equivalent to one year of the artificial hip joints in human body [15]. Then the running-in wear can be estimated by multiplying the wear volume in one second by one million. Results obtained in this way considering different clearances are shown in Fig. 6 (triangular dots) together with the experimental values reported by Dowson [15] (circular dots). Interestingly, the model predictions are very close to the experimental data. This confirms the validity of the model described by Eq. (11).

In order to simplify the calculation, average normal loads and velocities are commonly used in the quantitative assessment of loading and wear of artificial hip joints. In order to verify the accuracy of this simplification, the average wear rates were calculated using Eq. (11) by considering an average normal load of $1500 \mathrm{~N}$ and an angular velocity of $1.5 \mathrm{rad} / \mathrm{s}$. Calculated values are plotted in Fig. 6 with square dots. It can be found that the

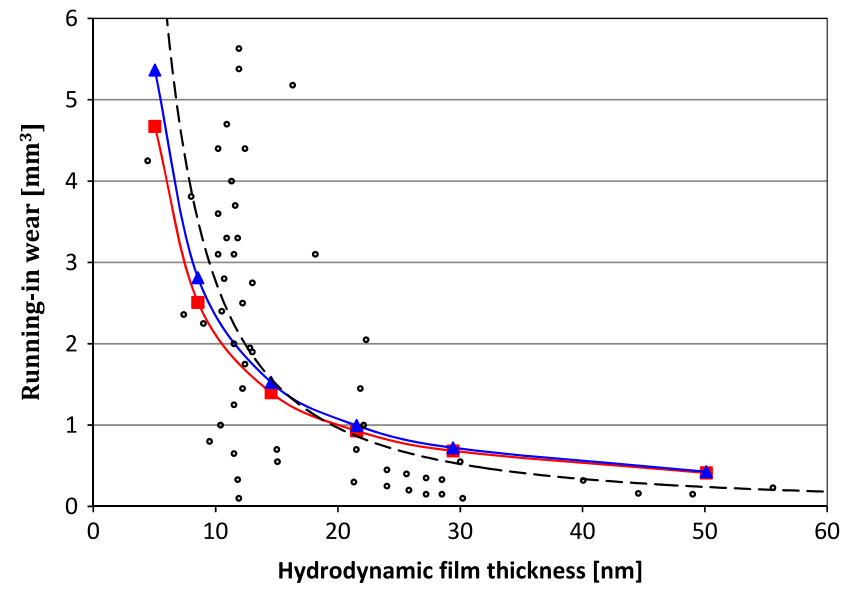

Fig. 6. The experimental running-in wear results (circular dots) with different film thicknesses and fitting line (dashed line) from [15] and model predicted values from integral of instantaneous wear rate (triangular dots and interpolation) and using average load and velocity (square dots and interpolation) ( $R=18 \mathrm{~mm}$, $c_{\mathrm{R}}=200 \mu \mathrm{m}, 100 \mu \mathrm{m}, 50 \mu \mathrm{m}, 30 \mu \mathrm{m}, 20 \mu \mathrm{m}$ and $10 \mu \mathrm{m}$, successively).

calculated values using average normal load and angular velocity can sufficiently precisely represent the real running-in wear. So, for the following calculations of the wear rate of artificial hip joints, in order to simplify the mathematical work, average normal load and angular velocity are systematically used.

\subsection{The effect of the parameters}

The composite model described by Eq. (11) allows assessing the role of a number of parameters on the running-in wear rate. For this, the variation of the mechanical, chemical and total wear rate were calculated as a function of normal load, velocity, head radius and radial clearance and plotted in Fig. 7.

Default values for calculation were: $1500 \mathrm{~N}$ normal force, $1.5 \mathrm{rad} / \mathrm{s}$ angular velocity, $18 \mathrm{~mm}$ head radius, $30 \mu \mathrm{m}$ radial clearance, CoCrMo alloy (Young's modulus $248 \mathrm{GPa}$, Poisson's ratio 0.3), $0.001 \mathrm{~Pa} \cdot \mathrm{s}$ fluid viscosity, $700 \mathrm{HV}$ micro surface hardness, $1 \mathrm{mC} / \mathrm{cm}^{2}$ passivation charge density. Fig. $7 \mathrm{a}$ and $\mathrm{b}$ show the influence of normal force and angular velocity on the wear rate under simulator relevant conditions. It can be seen that both the mechanical and chemical wear rate increase with the increase of normal force, which can be explained by the larger contact pressure resulting in severer plastic deformation of contact asperities and reduction of hydrodynamic film thickness. Interestingly, chemical and mechanical wear contribute in similar proportions to the overall wear. Note that the proportion varies with load, with chemical wear prevailing mainly at lower loads. Above approximately $1000 \mathrm{~N}$ the total wear varies nearly linearly with load. This trend is in agreement with experimental observations from simulators $[10,11]$. Fig. $7 \mathrm{~b}$ indicates that velocity has little effect on the mechanical wear but great influence on the chemical wear. High velocity increases the hydrodynamic film thickness (Eq. 7), thus alleviating the plastic deformation of the asperities, but it also increases the sliding distance per unit time, increasing the amount of material degradation. In the case of mechanical wear, these two effects compensate each other while in the case of chemical wear, lubrication cannot compensate the increasing depassivation rate caused by the raising velocity.

Fig. 7c and d show the variation of wear rate with the increase of head radius and radial clearance. Larger radius or smaller clearance gives lower wear rate essentially because both parameters lead to larger effective radius. Interestingly, mechanical wear decreases steadily with increasing head radius. This is not the case of chemical wear that, according to the model, is much less affected by the head radius. As a consequence, above approximately $20 \mathrm{~mm}$ radius, the total wear is 
a

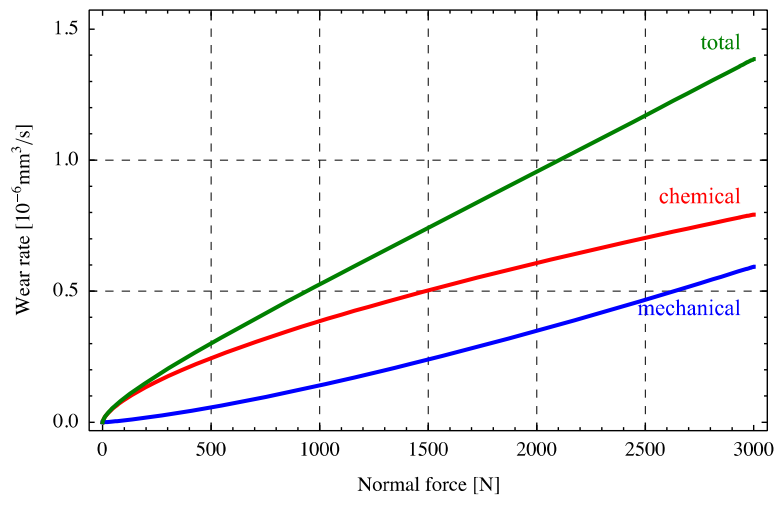

C

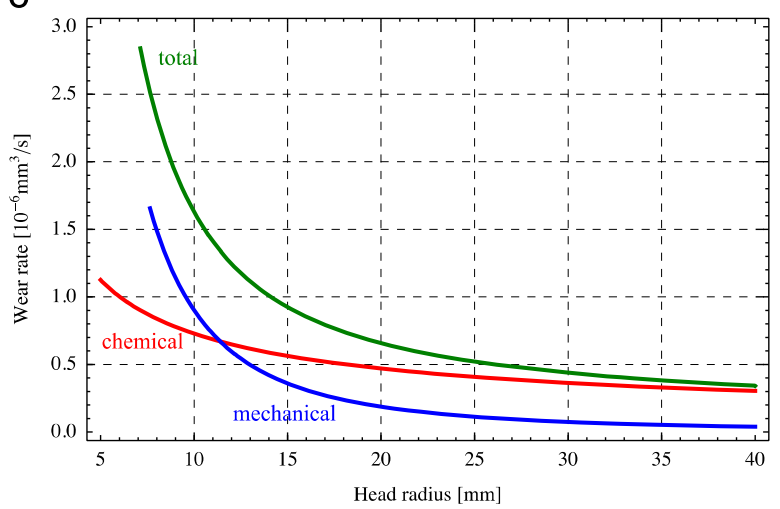

b

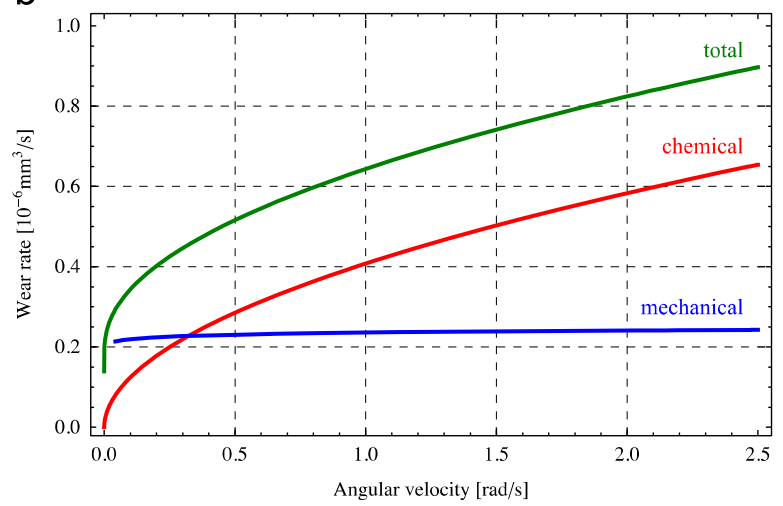

d

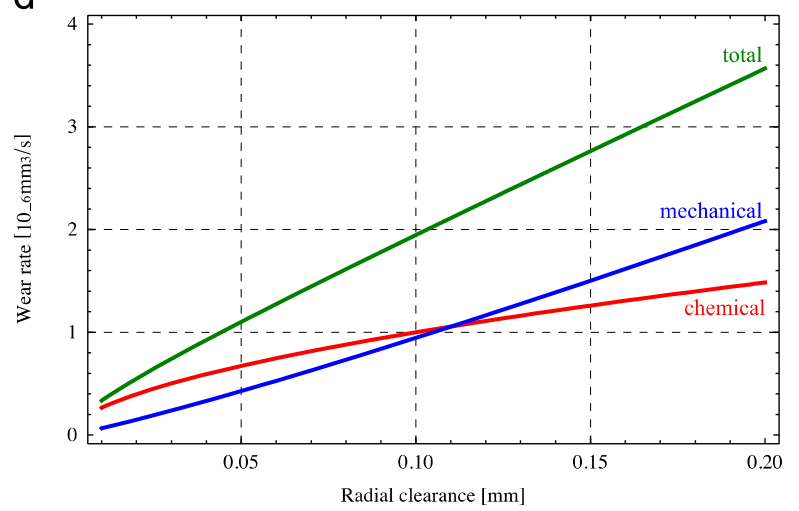

Fig. 7. The variation of mechanical, chemical and total wear rate with normal force, angular velocity, head radius and radial clearance under simulator conditions.

dominated by chemical wear and thus increasing the head radius does not imply significant reduction in material damage despite the enhanced hydrodynamic lubrication. This model prediction agrees well with literature reports [12], which found that large head radius do not necessarily provide lower wear. Smaller clearance reduces the wear rate dramatically, which corresponds to the former studies [12,35].

\subsection{Wear rate predictions for different material couples}

CoCrMo alloys can be used in hip joints as self mated contact couple (MoM joint) or in contact with ultra high molecular weight polyethylene (UHMWPE) (MoP joint) or alumina based ceramics (CoM contact). The model was therefore applied to such contacts in order to evaluate the degradation of the CoCrMo alloy in different material couples. Note that in the case of MoP joints, wear of the UHMWPE is much larger than that of metal and determines lifetime of the implant. The default values mentioned in the previous paragraph were used for calculation in combination with Young's moduli of $1 \mathrm{GPa}$ and $350 \mathrm{GPa}$ and Poisson's ratios of 0.4 and 0.22 for UHMWPE and Alumina, respectively. The calculated values are listed in Table 3 .

Not surprisingly, the model predicts lower wear rates of CoCrMo alloy for the softer MoP couple due to the lower contact pressure and the thicker hydrodynamic film (Eq. (7)). For the same reason, wear appears slightly more elevated in CoM compared to MoM. Interestingly, in the MoP contact chemical wear predominates (90\% of the overall wear) while its contribution is less (67\%) in MoM and CoM contacts.

The estimated wear of the MoP contact constitutes most likely an upper limit. Indeed, due to the large compliance of the polymer, the local pressure on asperities may become very small and, depending on local contact pressure, lower than the critical threshold needed for plastic deformation. Diomidis et al. [36]
Table 3

Wear rate of CoCrMo alloy in different material coupled artificial hip joints $\left(R=18 \mathrm{~mm}, c_{\mathrm{R}}=30 \mu \mathrm{m}\right)$.

\begin{tabular}{llll}
\hline \multirow{2}{*}{ Material couple } & \multicolumn{3}{l}{ Wear rate of the CoCrMo alloy $\left[\mathrm{mm}^{3} / \mathrm{M}-\mathrm{cycle}\right]$} \\
\cline { 2 - 4 } & Mechanical & Chemical & Total \\
\hline MoP & 0.011 & 0.106 & 0.117 \\
MoM & 0.239 & 0.503 & 0.742 \\
CoM & 0.262 & 0.526 & 0.788 \\
\hline
\end{tabular}

Young's modulus and Poisson's ratio of typical artificial hip joint materials: UHMWPE ( $E=1 \mathrm{GPa}, v=0.4$ ), CoCrMo ( $E=248 \mathrm{GPa}, v=0.3), \mathrm{Al}_{2} \mathrm{O}_{3}(E=350 \mathrm{GPa}$, $v=0.22$ ).

reported the existence of such a threshold for $\mathrm{Ti}$ alloys at approximately $20 \mathrm{MPa}$. Asperities loaded below the critical threshold are not expected to further contribute to wear and depassivation. However, the model does not contemplate a critical pressure threshold and therefore likely over estimates wear of MoP contacts.

\section{Discussion}

The proposed model is based on a simple mechanistic interpretation of tribocorrosion. Nevertheless it permits to predict with a remarkable precision the wear rates observed in tribometers as well as in hip joint simulators. Furthermore, it permits to identify the dominating wear mechanism (chemical, mechanical) and to evaluate the influence of well-defined parameters. For example, the model indicates that wear accelerated corrosion is a major contribution to overall wear of hip joints, while predictions based on one single mechanism may lead erroneous conclusions since 
chemical wear and mechanical wear depends on different combinations of parameters. As shown in Fig. 7, moving to larger head sizes appears, according to pure mechanical considerations, as an effective method to significantly reduce wear. Nevertheless, considering chemical wear, this parameter has a much smaller impact.

For quantitative predictions, the model requires calibration of the $k_{\text {mech }}$ and $k_{\text {chem }}$ factors as well as the input of materials specific parameters such as passivation charge density and surface hardness. In the present case (CoCrMo alloys, aqueous solutions) the use of a simple laboratory triboelectrochemical set-up consisting of a smooth alumina ball sliding against a polished CoCrMo flat surface appears as a suitable and simple method. Note that plastic deformation dominates the metal response to friction in the calibration system [25], which thus satisfy the underlying hypotheses of the model, that is, plastic deformation at asperity contacts. Systems involving other wear mechanisms such as brittle fracture or subsurface fatigue may not be appropriate for calibration.

The passivation charge density is a crucial parameter affecting chemical wear. As shown in Fig. 3, its value depends on the electrode potential. The correct input of the passivation charge density requires the knowledge of the electrode potential of the contacting metal, a value that need to be experimentally determined for each combination of metal and electrolyte as well as other critical parameters affecting the potential such as temperature and mass transport conditions in the fluid. Furthermore, the potential may change during tribocorrosion tests [9,25]. Further, chemical species present in the electrolyte may modify the passivation charge density. For example, chelating agents such as lactic acid and phosphate ions were found to modify the passivation mechanisms and thus the passivation charge density of tungsten in acidic media [37]. Note also that the measurement of the passivation charge density is not trivial and the determined values may vary depending on the method used for measuring it [22]. So, the same method used for calibration should be used to determine the $Q_{p}$ in the system under investigation.

During rubbing, metals usually undergo work hardening resulting in a hardness gradient from the surface to the bulk material [17]. The degree of hardening being difficult to predict, experimental determination of hardness is needed. The presence of the gradient implies that the measured hardness changes with indentation load. Thus, the same indentation load applied for calibration (Fig. 2) should be used for measuring the surface hardness of the investigated system.

The present model suffers of some limitations linked to the empirical nature of Eq. (6) that relates average wear during the running-in period to the hydrodynamic film thickness. This equation is issued from a set of experimental wear investigation of CoCrMo MoM hip joints and is therefore specific to the mixed lubrication conditions found there, in particular the surface roughness. Indeed, mixed lubrication establishes when the ratio between asperity heights, as characterised by the $R_{\mathrm{a}}$ value, and hydrodynamic film thickness ranges between 1 and 3 . The Dowson's equation is in principle valid for the specific roughness conditions found in hip joints characterised by $R_{\mathrm{a}}$ values of typically a few ten nanometres, that is, satisfying the condition for mixed lubrication for the typical hydrodynamic film thicknesses established in hip joints. For smoother or rougher surfaces the applicability of Dowson's equation needs to be verified. Indeed, Stemp et al. [38] observed that during tribocorrosion of stainless steel the extent of mechanical and chemical wear was significantly affected by the roughness of the counterpart.

As another limitation, the model ignores time dependent effects. As a consequence of wear, the asperity height and thus the surface roughness are expected to steadily decrease with sliding time. This implies that a larger fraction of the load is progressively carried out by the hydrodynamic film to the detriment of the load transmitted by asperities contacts. The wear rate should correspondingly decrease with time. This is indeed observed in hip joint simulator where, after an initial high wear phase (run-in period), the wear rate becomes smaller and even negligible (steady state period). The build up of third bodies during rubbing also affects the load carried out by asperities. In artificial hip joints, the formation of carbonaceous tribolayer [8] and the deposition of agglomerated proteins [13] have been reported. These phenomena are expected to reduce the asperity load and thus wear. The formation kinetics of third bodies and their effect on load distribution and hydrodynamic lubrication should therefore be considered in further model improvements.

\section{Conclusions}

- A tribocorrosion model for passive metals undergoing plastic deformation at asperity contacts combining mechanical wear (Archard's law), chemical wear (wear accelerated corrosion) and hydrodynamic lubrication was proposed to quantitatively describe and predict material damage. The model permits to identify the dominating wear mechanism and to evaluate the influence of well-defined mechanical, electrochemical, material and lubricant parameters.

- As applied to CoCrMo sliding tribocorrosion contacts, the model predicts remarkably well wear rates observed in tribometers and the running-in wear rate of artificial hip joints tested in simulators.

- In the case of CoCrMo MoM hip joints, the model predictions concerning the effect of parameters such as normal load, head radius and clearance closely correlate with experimental observations.

- According to the model, both mechanical wear and chemical wear significantly contribute to hip joint degradation. Thus predictions based on only one mechanism likely lead to erroneous conclusions. This is for example the case of head radius that while having a significant effect on mechanical wear little affects chemical wear. The model also predicts that MoM hip joint damage occurs also during the swing phase of a single gait cycle and this is because of the large contribution of chemical wear.

\section{Acknowledgements} Council.

Shoufan Cao acknowledges grant from the China Scholarship

\section{References}

[1] S. Kurtz, K. Ong, E. Lau, F. Mowat, M. Halpern, Projections of primary and revision hip and knee arthroplasty in the United States from 2005 to 2030, J. Bone Joint Surg. Am 89 (4) (2007) 780-785.

[2] L. Mattei, F. Di Puccio, B. Piccigallo, E. Ciulli, Lubrication and wear modelling of artificial hip joints: A review, Tribol. Int. 44 (5) (2011) 532-549.

[3] A. Neville, Y. Yan, Biotribocorrosion: surface interactions in total joint replacement (TJR), in: D. Landolt, S. Mischler (Eds.), Tribocorrosion of Passive Metals and Coatings, Woodhead Publishing Limited, Cambridge, UK, 2011, pp. 337-367

[4] I. Catelas, J.B. Medley, P.A. Campbell, O.L. Huk, J.D. Bobyn, Comparison of in vitro with in vivo characteristics of wear particles from metal-metal hip implants, J. Biomed. Mater. Res. B 70B (2) (2004) 167-178.

[5] P.M. Whittingham-Jones, E. Dunstan, H. Altaf, S.R. Cannon, P.A. Revell, T.W. R. Briggs, Immune responses in patients with metal-on-metal hip articulations, J. Arthroplasty 23 (8) (2008) 1212-1218.

[6] M. Huber, G. Reinisch, G. Trettenhahn, K. Zweymuller, F. Lintner, Presence of corrosion products and hypersensitivity-associated reactions in periprosthetic tissue after aseptic loosening of total hip replacements with metal bearing surfaces, Acta Biomater. 5 (1) (2009) 172-180. 
[7] G.M. Keegan, I.D. Learmonth, C.P. Case, Orthopaedic metals and their potential toxicity in the arthroplasty patient, J. Bone Joint Surg. Br. 89-B (5) (2007) 567-573.

[8] M.A. Wimmer, C. Sprecher, R. Hauert, G. Tager, A. Fischer, Tribochemical reaction on metal-on-metal hip joint bearings: A comparison between in-vitro and in-vivo results, Wear 255 (7-12) (2003) 1007-1014.

[9] S. Mischler, A. Igual Munoz, Wear of CoCrMo alloys used in metal-on-metal hip joint: a tribocorrosion appraisal, Wear 297 (1-2) (2013) 1081-1094.

[10] F. Liu, I. Leslie, S. Williams, J. Fisher, Z. Jin, Development of computational wear simulation of metal-on-metal hip resurfacing replacements, J. Biomech. 41 (3) (2008) 686-694.

[11] M.S. Uddin, L.C. Zhang, Predicting the wear of hard-on-hard hip joint prostheses, Wear 301 (1-2) (2013) 192-200.

[12] C.B. Rieker, R. Schon, R. Konrad, G. Liebentritt, P. Gnepf, M. Shen, P. Roberts, P. Grigoris, Influence of the clearance on in-vitro tribology of large diameter metal-on-metal articulations pertaining to resurfacing hip implants, Orthop. Clin. N. Am 36 (2) (2005) 135-142.

[13] C. Myant, P. Cann, On the matter of synovial fluid lubrication: Implications for metal-on-metal hip tribology, J. Mech. Behav. Biomed 34 (2014) 338-348.

[14] D. Dowson, The relationship between steady-state wear rate and theoretica film thickness in metal-on-metal total replacement hip joints, in: D. Dowson, M. Priest, G. Dalmaz, A.A. Lubrecht (Eds.), Tribological Research and Design for Engineering Systems, Elsevier, Leeds, UK, 2003, pp. 273-280.

[15] D. Dowson, Tribological principles of metal-on-metal hip joint design, P. I. Mech. Eng. H 220 (2) (2006) 161-171.

[16] D. Dowson, C. Hardaker, M. Flett, G.H. Isaac, A hip joint simulator study of the performance of metal-on-metal joints: part II: design, J. Arthroplasty 19 (8) (2004) 124-130.

[17] R. Büscher, A. Fischer, The pathways of dynamic recrystallization in all-metal hip joints, Wear 259 (7-12) (2005) 887-897.

[18] A.W. Hodgson, S. Mischler, B. Von Rechenberg, S. Virtanen, An analysis of the in vivo deterioration of Co-Cr-Mo implants through wear and corrosion, P. I. Mech. Eng. H 221 (H3) (2007) 291-303.

[19] A. Igual Munoz, N. Espallargas, Tribocorrosion mechanisms in sliding contacts, in: D. Landolt, S. Mischler (Eds.), Tribocorrosion of Passive Metals and Coatings, Woodhead Publishing Limited, Cambridge, UK, 2011, pp. 118-152.

[20] H.H. Uhlig, Mechanism of fretting corrosion, J. Appl. Mech. 21 (1954) 401-407.

[21] S. Mischler, Triboelectrochemical techniques and interpretation methods in tribocorrosion: A comparative evaluation, Tribol. Int. 41 (7) (2008) 573-583.

[22] S. Mischler, S. Debaud, D. Landolt, Wear-accelerated corrosion of passive metals in tribocorrosion systems, J. Electrochem. Soc. 145 (3) (1998) 750-758.

[23] S. Mischler, A. Spiegel, M. Stemp, D. Landolt, Influence of passivity on the tribocorrosion of carbon steel in aqueous solutions, Wear 251 (1-12) (2001) 1295-1307.
[24] D. Landolt, S. Mischler, M. Stemp, Electrochemical methods in tribocorrosion: a critical appraisal, Electrochim. Acta 46 (24-25) (2001) 3913-3929.

[25] S. Guadalupe Maldonado, S. Mischler, M. Cantoni, M. Cantoni, W-J. Chitty, C. Falcand, D. Hertz, Mechanical and chemical mechanisms in the tribocorrosion of a stellite type alloy, Wear 308 (1-2) (2013) 213-221.

[26] B. Hamrock, D. Dowson, Elastohydrodynamic lubrication of elliptical contacts for material of low elastic modulus I-fully flooded conjunction, J. Lubr. Technol 100 (2) (1978) 236-245.

[27] M. Azzi, J.-E. Klemberg-Sapieha, Tribocorrosion test protocols for sliding contacts, in: D. Landolt, S. Mischler (Eds.), Tribocorrosion of Passive Metals and Coatings, Woodhead Publishing Limited, Cambridge, UK, 2011, pp. 222-238.

[28] J.A. Bardwell, G.I. Sproule, M.J. Graham, Ex situ surface analysis of passive films on Fe-Cr alloys, J. Electrochem. Soc. 140 (1) (1993) 50-53.

[29] J.A. Bardwell, G.I. Sproule, D.F. Mitchell, B. Macdougall, M.J. Graham, Nature of the passive film on $\mathrm{Fe}-\mathrm{Cr}$ alloys as studied by ${ }^{18} \mathrm{O}$ secondary ion mass spectrometry: reduction of the prior film and stability to ex situ surface analysis, J. Chem. Soc. Faraday T. 87 (7) (1991) 1011-1019.

[30] A.W.E. Hodgson, S. Kurz, S. Virtanen, V. Fervel, C.-O.A. Olsson, S. Mischler, Passive and transpassive behaviour of CoCrMo in simulated biological solutions, Electrochim. Acta 49 (13) (2004) 2167-2178.

[31] A. Igual Munoz, L. Casabán Julián, Influence of electrochemical potential on the tribocorrosion behaviour of high carbon CoCrMo biomedical alloy in simulated body fluids by electrochemical impedance spectroscopy, Electrochim. Acta 55 (19) (2010) 5428-5439.

[32] L. Casabán Julián, A. Igual Munoz, Influence of microstructure of HC CoCrMo biomedical alloys on the corrosion and wear behaviour in simulated body fluids, Tribol. Int. 44 (3) (2011) 318-329.

[33] Y. Yan, A. Neville, D. Dowson, S. Williams, J. Fisher, Effect of metallic nanoparticles on the biotribocorrosion behaviour of metal-on-metal hip prostheses, Wear 267 (5-8) (2009) 683-688.

[34] J. Hesketh, X. Hu, Y. Yan, D. Dowson, A. Neville, Biotribocorrosion: Some electrochemical observations from an instrumented hip joint simulator, Tribol. Int. 59 (2013) 332-338.

[35] F.W. Chan, J.D. Bobyn, J.B. Medley, J.J. Krygier, M. Tanzer, Wear and lubrication of metal-on-metal hip implants, Clin. Orthop. Relat. R 369 (1999) 10-24.

[36] N. Diomidis, S. Mischler, N.S. More, M. Roy, Tribo-electrochemical characterization of metallic biomaterials for total joint replacement, Acta Biomater. 8 (2) (2012) 852-859.

[37] J. Stojadinovic, D. Bouvet, M. Declercq, S. Mischler, Influence of chelating agents on the tribocorrosion of tungsten in sulphuric acid solution, Electrochim. Acta 56 (20) (2011) 7131-7140.

[38] M. Stemp, S. Mischler, D. Landolt, The effect of mechanical and electrochemical parameters on the tribocorrosion rate of stainless steel in sulphuric acid, Wear 255 (1-6) (2003) 466-475. 